\title{
Modelling free surface flow with curvilinear streamlines by a non-hydrostatic model
}

\author{
Yebegaeshet T. Zerihun
}

David \& James - Engineering and Environmental Consultancy, 204 Albion Road, Victoria 3350, Australia. Tel.: +61(3) 5331 6747. E-mail: zyebegaeshet@gmail.com

\begin{abstract}
This study addresses a particular phenomenon in open channel flows for which the basic assumption of hydrostatic pressure distribution is essentially invalid, and expands previous suggestions to flows where streamline curvature is significant. The proposed model incorporates the effects of the vertical curvature of the streamline and steep slope, in making the pressure distribution non-hydrostatic, and overcomes the accuracy problem of the Saint-Venant equations when simulating curvilinear free surface flow problems. Furthermore, the model is demonstrated to be a higher-order one-dimensional model that includes terms accounting for wave-like variations of the free surface on a constant slope channel. Test results of predicted flow surface and pressure profiles for flow in a channel transition from mild to steep slopes, transcritical flow over a short-crested weir and flow with dual free surfaces are compared with experimental data and previous numerical results. A good agreement is attained between the experimental and computed results. The overall simulation results reveal the satisfactory performance of the proposed model in simulating rapidly varied gravity-driven flows with predominant non-hydrostatic pressure distribution effects. This study suggests that a higher-order pressure equation should be used for modelling the pressure distribution of a curvilinear flow in a steeply sloping channel.
\end{abstract}

Keywords: Curvilinear flow; Finite difference method; Hydraulic structures; Hydrodynamics; Non-hydrostatic pressure; Nonuniform flow.

\section{INTRODUCTION}

The prediction of free surface flows using numerical models is of great interest to researchers and practising engineers. In many problems of practical interest such as flow over a spillway, numerical modelling can be more attractive than physical model tests in terms of cost and time. Furthermore, the detailed description of the flow field can be predicted for the entire flow domain with a sufficient accuracy. Most free surface flows are well described by the Saint-Venant equations. However, the depth averaging process used to develop these equations in order to fulfil the assumptions of mean velocity and hydrostatic pressure distributions sacrifices the necessary flow details over the vertical dimension. Consequently, the equations represent the lowest-order in approximation and cannot retain accuracy for flow situations where the effects of non-hydrostatic pressure distribution are significant and essential (Steffler and Jin, 1993). In many practical problems such as transcritical flow in open channels, the flow field is characterised by a departure from hydrostatic distribution of pressure (Castro-Orgaz and Chanson, 2014; Zerihun and Fenton, 2006). For such flow problems, the dynamic pressure due to the curvature of streamline needs to be accounted for properly in order to recover more vertical details. In engineering practice, rapidly varied flows in hydraulic structures, free overfall and flow over bed forms are examples of flows that require the application of relatively accurate methods for exact description of the flow field.

Attempts have been made in the past to model curvilinear free surface flow problems using different approaches. Most of these attempts assumed that the flow could be approximated as potential flow in which variable velocity and/or non-hydrostatic pressure fields are incorporated into the energy and momentum equations following the Boussinesq approximation (see e.g., Castro-Orgaz, 2013; Castro-Orgaz and Hager, 2013; CastroOrgaz and Hager, 2014; Matthew, 1991). Zerihun (2004) aptly indicated the links between the different forms of Boussinesqtype equations proposed earlier, and described thoroughly the impact of the special assumptions made by the authors on the application of these equations. Dressler (1978) derived generalised, nonlinear, shallow-water equations which explicitly include bottom curvature effects. In an analogous manner, Berger and Carey (1998), Dowels et al. (2006) and Anh and Hosoda (2007) extended the Dressler approach and developed the depth-averaged equations for modelling free surface flows over curved boundaries. However, all these equations do not include terms accounting for free surface curvature and apply only to open channel flows in which the effect of bottom curvature is significant. Other investigators (Steffler and Jin, 1993) developed the vertically averaged and moment equations for the case of one-dimensional model in which they applied the moment of momentum principle for computing the unspecified parameters in a priori assumed velocity and pressure distributions. In addition, the Blom and Booij's (1995) modified shallow-water equations and the multilayer depth-averaged flow models have been used to overcome the hydrostatic pressure hypothesis. The recent application of the latter approach used the moment principle along with the assumption of linear distributions of velocity and pressure in each layer to develop the layer-averaged equations (Xia and Jin, 2006). These multilayer models, however, require many layers of small vertical thickness for accurate description of the flow field. As a consequence, computational time becomes significant. More recently, Zobeyer and Steffler (2012) modelled the two-dimensional (2-D) vertical plane flows by coupling the one-dimensional depth-averaged equations with the Reynolds-averaged Navier-Stokes equations. Similar to Steffler and Jin's approach, this method presents complex system of equations, reducing the efficiency and stability of the numerical model.

Fenton (1996) presented a method to model flow problems with substantial curvature of streamline. He used the momen- 
tum principle along with the assumption of a constant dynamic pressure correction term across a vertical section to develop the equations. The method allows easy incorporation of the free surface and bed curvature effects, and thus better representation of the effects of non-hydrostatic pressure and/or nonuniform velocity distributions. Even though the pressure equation accounts for the effect of the vertical acceleration, its application is limited to the prediction of pressure distribution for moderately curved flow problems with single free surface (Zerihun and Fenton, 2006). This indicates the need for modifying the Fenton's method for developing a general purpose flow model. It is the aim of this work to generalise this method for developing a one-dimensional non-hydrostatic flow model that incorporates a higher-order correction not only for the effect of the dynamic pressure due to the vertical curvature of the streamline but also for the effect of channel steep slope. Compared to previous governing equations, the proposed model is capable of providing a better description of the curved flow situations and is not restricted to irrotationality. The study also aims to investigate the suitability of this model for a wide variety of practical curvilinear free surface flow problems. These include flows in a channel transition from mild to steep slopes; transcritical flows over short-crested weirs; and flow with dual free surfaces. The three case studies are ideal for examining the performance of the flow model because each of them has different characteristics and requires completely different techniques to simulate them correctly. For all cases, the present model simulates the free surface as a shear-free undisturbed curved surface.

The paper begins with the derivation of the generalised higher-order equations, a brief depiction of the main features of the computational model, namely the spatial discretisation of the equations and boundary conditions, and an outline of the solution procedure for the resulting nonlinear discretised equations. Subsequently, the comparison of the computational results with experimental data and previous numerical results from the literature is presented. The paper concludes with a summary and discussion.

\section{MATHEMATICAL MODEL}

For an arbitrary channel section, Cartesian coordinates such that $x$ is horizontally along the channel, $y$ is horizontally in the transverse direction and $z$ is vertically upward are defined as illustrated in Figure 1. This figure also shows the streamline coordinate system with axes along and perpendicular to the streamline. The Fenton's (1996) one-dimensional dynamic equation for free surface flow, neglecting lateral flows, can be written as

$$
\frac{\partial Q}{\partial t}+\beta \frac{\partial}{\partial x}\left(\frac{Q^{2}}{A}\right)+\frac{1}{\rho} \int_{A} \frac{\partial p}{\partial x} d A+g A S_{f}=0,
$$

where $S_{f}$ denotes the friction slope, calculated from the Manning's equation or smooth boundary resistance law; $Q$ is the discharge; $A$ is flow cross-sectional area; $\rho$ is the density of the fluid; $p$ is the pressure; $\beta$ refers to the Boussinesq coefficient; $g$ is gravitational acceleration and $t$ is the time.

For a curvilinear flow with normal acceleration $a_{n}\left(=\kappa u^{2} / \cos ^{2} \theta\right)$, the equation for the pressure distribution can be derived by integrating the Euler's equation. Neglecting the temporal acceleration which is comparatively small, the

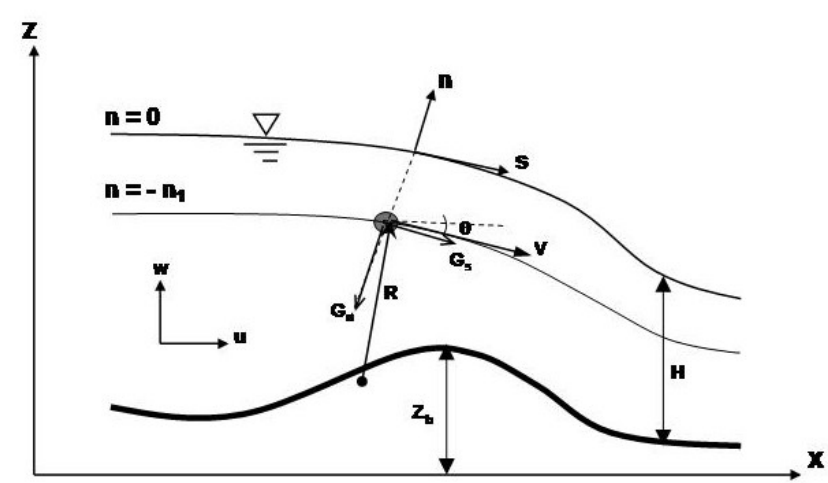

Fig. 1. Definition sketch for a curved bed channel and variables used in the model.

Euler's equation along the axis normal to the streamline can be written as (see e.g., White, 2003),

$$
\frac{1}{\rho} \frac{\partial p}{\partial n}=G_{n}-\lambda \kappa \frac{u^{2}}{\cos ^{2} \theta},
$$

where $u$ is the local horizontal flow velocity; $\theta$ is the streamline angle with the horizontal; $\kappa=1 / R$ is the local streamline curvature; $\partial p / \partial n$ is the pressure gradient; $\lambda$ is a factor related to the deviation of the free surface slope from the local streamline inclination; $R$ is the radius of curvature and $G_{n}=-g \cos \theta_{s}$. Following Serre's (1953) approach, the horizontal velocity component is approximated by its mean value. Although one can employ another vertical profile (e.g., linear, logarithmic-law, power-law, etc.) for horizontal velocities, the development of the governing equations becomes mathematically more cumbersome. As pointed out by Ghamry and Steffler (2002), such types of simplifying assumption for velocity distributions do not have significant impact on the model results for global flow characteristics. Also, the contributions of the transverse velocity components and the effects of the horizontal curvature of the streamline and the bed curvature across the channel are neglected. These are reasonable approximations especially for curvilinear free surface flow problems where the horizontal curvatures of the streamline are very small compared to its vertical curvatures.

The analysis of rapidly varied flow problems requires a closure hypothesis for the distributions of the dynamic pressure correction term, $\omega$ across a vertical section. A linear variation of $\omega$ from the bed to the free surface is assumed. After substituting the relationship $\partial n=\partial z / \sqrt{1+\left(\eta_{x}\right)^{2}}$ in Equation (2) and rearranging the terms, the resulting distribution equation then becomes

$\omega=\frac{\lambda}{\sqrt{1+\left(z_{x}\right)^{2}}} \frac{\partial^{2} z}{\partial x^{2}}=\omega_{b}+\left(\frac{z-Z_{b}}{\eta-Z_{b}}\right)\left(\omega_{s}-\omega_{b}\right)$,

where $\eta$ and $Z_{b}$ are elevations of the free surface and bed respectively and $z$ is vertical elevation of a point in the flow field. This equation provides a continuous variation between the boundary values of the dynamic pressure correction term. The subscripts $b$ and $s$ refer to bed and free surface conditions respectively. Inserting Equation (3) into Equation (2) and verti- 
cally integrating from $z$ to $\eta$ yields the pressure equation in the preferred Cartesian coordinate system for flow in a rectangular channel

$$
\begin{aligned}
& \frac{p}{\rho}=\frac{g(\eta-z)}{1+\left(\eta_{x}\right)^{2}}+\frac{Q^{2} \omega_{b}(\eta-z)}{B^{2} H^{2} \sqrt{1+\left(\eta_{x}\right)^{2}}} \\
& +\frac{Q^{2}}{B^{2} H^{2} \sqrt{1+\left(\eta_{x}\right)^{2}}}\left(\frac{\omega_{s}-\omega_{b}}{\eta-Z_{b}}\right)\left(\left(\frac{\eta^{2}-z^{2}}{2}\right)-Z_{b}(\eta-z)\right) .
\end{aligned}
$$

At the free surface and bed, the dynamic pressure correction terms are expressed by the following relations from Equation (3):

$\lambda_{b}=\frac{1}{\sqrt{1+\left(Z_{b}^{\prime}\right)^{2}} \sqrt{1+\left(\eta_{x}\right)^{2}}}+\frac{\eta_{x} Z_{b}^{\prime}}{\sqrt{1+\left(Z_{b}^{\prime}\right)^{2}} \sqrt{1+\left(\eta_{x}\right)^{2}}}$

$\omega_{s}=\frac{\eta_{x x}}{\sqrt{1+\left(\eta_{x}\right)^{2}}}$

$\omega_{b}=\frac{Z_{b}^{\prime \prime}}{\left(1+\left(Z_{b}^{\prime}\right)^{2}\right) \sqrt{1+\left(\eta_{x}\right)^{2}}}$.

Substituting Equations (6) and (7) in Equation (4) gives,

$$
\begin{aligned}
& \frac{p}{\rho g}=\frac{(\eta-z)}{\Omega_{1}}+\frac{Q^{2} Z_{b}^{\prime \prime}(\eta-z)}{g B^{2} H^{2} \Omega_{1} \Omega_{2}} \\
& +\frac{Q^{2}}{g B^{2} H^{2}}\left(\frac{\eta_{x x}}{\Omega_{1}}-\frac{Z_{b}^{\prime \prime}}{\Omega_{1} \Omega_{2}}\right)\left(\left(\frac{\eta^{2}-z^{2}}{2\left(\eta-Z_{b}\right)}\right)-\left(\frac{Z_{b}(\eta-z)}{\eta-Z_{b}}\right)\right),
\end{aligned}
$$

where $\eta$ is the free surface elevation; $\Omega_{1}=1+\left(\eta_{x}\right)^{2}$ and $\Omega_{2}=1+\left(Z_{b}^{\prime}\right)^{2}$. In the above equations, the subscript $x$ denotes differentiation with respect to the streamwise horizontal axis.

Clearly the nature of the higher-order approximation can be identified; the pressure relation shows the normal hydrostatic pressure relation with correction factors as a result of the vertical acceleration and steep slope of the channel. It is noticed that the term which accounts for the dynamic effect due to the vertical curvatures of the streamline shows a quadratic variation in Equation (8). If the vertical curvatures of the streamline and the bed are neglected, i.e. $\partial^{2} \eta / \partial x^{2}=Z_{b}^{\prime \prime}=0$, Equation (8) reduces to the pressure distribution equation for flow in a steeply sloping channel (Chaudhry, 2008),

$$
\frac{p}{\rho g}=\frac{(\eta-z)}{\left(1+\left(\eta_{x}\right)^{2}\right)} \text {. }
$$

If Equation (8) is compared with the Castro-Orgaz and Hager's (2009) equation, which was developed based on the Matthew's (1991) approach, it is noticed that the present equation incorporates a higher-order correction for modelling the pressure distribution of a curvilinear flow in a steeply sloping channel. For flow in a small slope channel, $1+\left(\eta_{x}\right)^{2} \cong 1.0$, Equation (9) reduces to the well-known hydrostatic pressure equation.

It is assumed that the free surface is horizontal in the transverse direction, such that the pressure is not a function of $y$. Differentiating the pressure distribution Equation (8) with respect to $x$, integrating across the channel and then substituting into Equation (1) yields the following equation for flow in a rectangular channel after some manipulation:

$$
\begin{aligned}
& \frac{\partial Q}{\partial t}+\left(\beta \frac{2}{H}+\frac{2 Z_{b}^{\prime \prime}}{3 \Omega_{1}}+\frac{Z_{b}^{\prime \prime}}{3 \Omega_{1} \Omega_{2}}+\frac{2}{3 \Omega_{1}} \frac{\partial^{2} H}{\partial x^{2}}\right) \frac{Q}{B} \frac{\partial Q}{\partial x} \\
& +\frac{Q^{2}}{3 B \Omega_{1}} \frac{\partial^{3} H}{\partial x^{3}}+\frac{Q^{2} Z_{b}^{\prime}}{2 B H \Omega_{1}} \frac{\partial^{2} H}{\partial x^{2}} \\
& +\left(\frac{g B H}{\Omega_{1}}-\frac{Q^{2} Z_{b}^{\prime \prime}}{3 B H \Omega_{1} \Omega_{2}}+\frac{Q^{2}}{3 B H \Omega_{1}} \frac{\partial^{2} \eta}{\partial x^{2}}-\beta \frac{Q^{2}}{B H^{2}}\right) \frac{\partial H}{\partial x} \\
& +\frac{Q^{2}}{B}\left(\frac{Z_{b}^{\prime \prime \prime}}{3 \Omega_{1}}+\frac{Z_{b}^{\prime \prime \prime}}{6 \Omega_{1} \Omega_{2}}\right)+\frac{Q^{2}}{2 H B}\left(\frac{Z_{b}^{\prime} Z_{b}^{\prime \prime}}{\Omega_{1} \Omega_{2}}+\frac{Z_{b}^{\prime} Z_{b}^{\prime \prime}}{\Omega_{1}}\right) \\
& +g B H\left(\frac{Z_{b}^{\prime}}{\Omega_{1}}+S_{f}\right)=0,
\end{aligned}
$$

where $H$ is the local flow depth measured vertically; $Z_{b}^{\prime}, Z_{b}^{\prime \prime}$ and $Z_{b}^{\prime \prime \prime}$ are the first, second and third derivatives of the bed profile respectively and $B$ is the width of the channel. In Fenton's (1996) method, the curvatures of the flow surface and the bed were approximated by the expressions for flow with weak curvature of streamlines. However, the present method demonstrates that this approximation is not really required to develop the equation.

For steady flow condition, $\partial A / \partial t=B \partial H / \partial t=\partial Q / \partial x=0$. Using these relationships, Equation (10) reduces to

$$
\begin{aligned}
& \frac{Q^{2}}{3 B \Omega_{1}} \frac{d^{3} H}{d x^{3}}+\frac{Q^{2} Z_{b}^{\prime}}{2 B H \Omega_{1}} \frac{d^{2} H}{d x^{2}} \\
& +\left(\frac{g B H}{\Omega_{1}}-\frac{Q^{2} Z_{b}^{\prime \prime}}{3 B H \Omega_{1} \Omega_{2}}+\frac{Q^{2}}{3 B H \Omega_{1}} \frac{d^{2} \eta}{d x^{2}}-\beta \frac{Q^{2}}{B H^{2}}\right) \frac{d H}{d x} \\
& +\frac{Q^{2}}{B}\left(\frac{Z_{b}^{\prime \prime \prime}}{3 \Omega_{1}}+\frac{Z_{b}^{\prime \prime \prime}}{6 \Omega_{1} \Omega_{2}}+\frac{Z_{b}^{\prime} Z_{b}^{\prime \prime}}{2 H \Omega_{1} \Omega_{2}}+\frac{Z_{b}^{\prime} Z_{b}^{\prime \prime}}{2 H \Omega_{1}}\right) \\
& +g B H\left(\frac{Z_{b}^{\prime}}{\Omega_{1}}+S_{f}\right)=0 .
\end{aligned}
$$

Equation (11) is a generalised higher-order equation for steady 2-D flow problems where the effects of both the vertical curvatures of the streamline and steep slope are significant and essential. Furthermore, the equation incorporates terms accounting for wave-like variations of the free surface that allow simulation of short waves on a constant slope channel $\left(Z_{b}^{\prime \prime \prime}=Z_{b}^{\prime \prime}=0\right)$.

For the case of weakly curved free surface flow with negligible curvature of streamlines in a constant slope channel, the free surface streamline and bed curvature terms vanish. Under 
this flow condition, this higher-order equation, Equation (10), reduces to the steep-slope shallow-water equation (Berger and Carey, 1998).

$$
\begin{aligned}
& \frac{\partial Q}{\partial t}+\left(\frac{g B H}{1+\left(\eta_{x}\right)^{2}}-\beta \frac{Q^{2}}{B H^{2}}\right) \frac{\partial H}{\partial x} \\
& +2 \beta \frac{Q}{H B} \frac{\partial Q}{\partial x}+g B H\left(\frac{Z_{b}^{\prime}}{\left(1+\left(\eta_{x}\right)^{2}\right)}+S_{f}\right)=0 .
\end{aligned}
$$

It can be shown that Equation (10) reduces asymptotically to the De Saint-Venant equation for a nearly horizontal flow situation where the curvature and slope of the streamline are insignificant, $1 / \kappa_{b}$ and $1 / \kappa_{s} \rightarrow \infty$. In this study, Equation (11) will be applied to simulate a wide variety of steady curvilinear free surface flow problems. The numerical solutions of the equation will be compared with measurements and previous numerical results. The results of validating the flow model for selected flow problems will provide a significant insight into how well the model will function in different rapidly varied flow situations. It is important to note that simulating the free surface as a freely deformable air-water interface of a two-phase flow is beyond the scope of this work.

\section{Boundary conditions}

The complete numerical solutions of the curved-streamlined flow problems using the model equation require three external boundary conditions to be specified at the inflow and outflow sections which are located in the flow region where the effects of non-hydrostatic pressure distribution are insignificant. For given depth and discharge at the inflow section, the corresponding free surface slope $S_{H}$, can be computed numerically using the gradually-varied flow equation,

$S_{H}=\frac{d H}{d x}=\frac{S_{0}-S_{f}}{1-\beta F^{2}}$,

where $F$ is the Froude number and $S_{0}$ is the bed slope. At the outflow section, the flow depth is specified as a boundary condition and remained unchanged during the computations. In the absence of measured data, a method based on a quasi-uniform flow condition is applied to compute this depth.

\section{NUMERICAL MODEL}

Analytical solution that satisfies the specified boundary conditions is not feasible for the above nonlinear differential equation. The finite difference approximations, which are simple to code and extensively used to solve nonlinear differential equations (e.g., Fuhrman et al., 2005; Liang et al., 2007), are used here for its numerical solutions. For the purpose of discretisation, Equation (11) can be represented by a simple general equation as

$$
\left(\frac{d^{3} H}{d x^{3}}\right)_{j}+\xi_{0, j}\left(\frac{d^{2} H}{d x^{2}}\right)_{j}+\xi_{1, j}\left(\frac{d H}{d x}\right)_{j}+\xi_{2, j}=0,
$$

where $\xi_{0, j}, \xi_{1, j}$ and $\xi_{2, j}$ are the nonlinear coefficients at node $j$ associated with the flow profile equation and are given by

$\xi_{0, j}=\frac{3 Z_{b, j}^{\prime}}{H_{j}}$

$\xi_{1, j}=\frac{3 g B_{j}^{2} H_{j}}{Q_{j}^{2}}-\frac{Z_{b, j}^{\prime \prime}}{H_{j} \Omega_{2, j}}+\frac{Z_{b, j}^{\prime \prime}}{H_{j}}+\left(\frac{1}{H} \frac{d^{2} H}{d x^{2}}\right)_{j}-\frac{3 \beta \Omega_{1, j}}{H_{j}^{2}}$

$\xi_{2, j}=Z_{b, j}^{\prime \prime \prime}+\frac{Z_{b, j}^{\prime \prime \prime}}{2 \Omega_{2, j}}+\frac{3 Z_{b, j}^{\prime} Z_{b, j}^{\prime \prime}}{2 H_{j} \Omega_{2, j}}+\frac{3 Z_{b, j}^{\prime} Z_{b, j}^{\prime \prime}}{2 H_{j}}$

$+\frac{3 g B_{j}^{2} H_{j}}{Q_{j}^{2}}\left(\Omega_{1, j} S_{f, j}+Z_{b, j}^{\prime}\right)$.

In order to reduce the truncation errors due to the approximate nature of the finite difference expressions, higher-order accurate finite difference formulas are employed to replace the derivative terms in Equation (14) (see, Ferziger and Peric, 2002). Hence, the upwind finite difference approximations for the first and third derivative terms and central difference for the second derivative term (Abramowitz and Stegun, 1972) are used to discretise this third-order differential equation. After simplifying the discretised equation and rearranging the terms, the equivalent finite difference equation reads as

$$
\begin{aligned}
& H_{j-2}\left(-6+\xi_{1, j} h^{2}\right)+H_{j-1}\left(18+6 \xi_{0, j} h-6 \xi_{1, j} h^{2}\right) \\
& +H_{j}\left(-18-12 \xi_{0, j} h+3 \xi_{1, j} h^{2}\right)+H_{j+1}\left(6+6 \xi_{0, j} h+2 \xi_{1, j} h^{2}\right) \\
& +6 \xi_{2, j} h^{3}=0,
\end{aligned}
$$

where $h$ is the step size. Since the flow depth at $j=0$ (inflow section) is known, the depth at the imaginary node $(j=-1)$ can be determined from the estimated free surface slope, $S_{H}$, at the inflow section. Using the discretised type of Equation (13) at the inflow section and the expanded form of Equation (16) at $j=0$, the following implicit finite difference equation is obtained for predicting the depth at $j=-1$ :

$$
\begin{aligned}
& H_{-1}\left(-18+6 \xi_{0,0} h\right)-H_{0}\left(12 \xi_{0,0} h\right)+H_{1}\left(18+6 \xi_{0,0} h\right) \\
& +6 S_{H}\left(-6 h+\xi_{1,0} h^{3}\right)+6 \xi_{2,0} h^{3}=0 .
\end{aligned}
$$

Equations (16) and (17) constitute the one-dimensional finite difference scheme corresponding to the third-order differential equation, which is to be solved numerically as a two-point boundary value problem. Generally, such problem must be solved by an iterative method, which starts from an initial estimate for the free surface position using the Bernoulli and continuity equations. Then, to simulate the flow surface profile, Equation (16) is applied at different nodal points within the solution domain and results in a sparse system of non-linear algebraic equations. These equations together with Equation (17), and the two boundary values specified at the inflow and outflow sections, are solved by the Newton-Raphson method 
with a numerical Jacobian matrix. The convergence of the solution is assessed by the following criterion:

$$
\left(\sum_{j=1}^{m}\left|\delta H_{j}\right| / \sum_{j=1}^{m}\left|H_{j}\right|\right) \leq \text { tolerance, }
$$

where $\delta H_{j}$ is the correction depth in any stage of the iteration process at nodal point $j$ and $m$ is the total number of nodes in the solution domain excluding extreme nodes at the inflow and outflow sections. In this study, a convergence tolerance of $10^{-6}$ is used.

For the solution of the pressure equation, a similar finite difference approximation is used to discretise the derivative term in Equation (8). Using the known flow depths from the solution of the flow profile equation, this discretised equation yields the bed pressure (after replacing $z$ by $Z_{b}$ ) at different nodal points.

\section{VALIDATION OF THE MODEL RESULTS}

The steady one-dimensional flow model, presented in the previous section, was used for simulating: i) curvilinear free surface flow in a channel transition from mild to steep slopes; ii) transcritical flow over a short-crested weir; iii) open channel flow with dual free surfaces. For all case studies, the experiments were performed in hydraulically smooth laboratory flumes which were made of plexiglass. For the simulation of flow over such boundaries, a smooth boundary resistance law, which was developed based on the Darcy-Weisbach equation and Haaland (1983) friction factor formula, was applied for computing the friction slope (Castro-Orgaz and Chanson, 2011; Zerihun, 2004). In this method, the effect of channel steep slope on flow resistance was accounted for by using a depth normal to the bed for the wetted perimeter term. For computational simplicity, $\beta$ was assumed as unity in the model. As noted by Zerihun (2004), such simplifying assumption does not cause any significant discrepancies between the predictions and the experimental data.

For all test cases studied, the size of the steps was designed to be fine enough to meet the requirements of accuracy and computational efficiency. The final computational results presented here were independent of the effect of spatial step size.

\section{Channel transition from horizontal to steep slopes}

The test results of Ehrenberger (1929) were invoked to test the ability of the model to simulate curvilinear-streamlined flow situations. The experiments were performed in a smooth channel $402 \mathrm{~mm}$ wide with a circular-shaped transition profile of radius $100 \mathrm{~mm}$ connecting the horizontal and the steep channels. Two steep slopes of $45^{\circ}$ and $60^{\circ}$ were studied. Flow surface and bed pressure profiles were measured for a discharge of 20,40 and $60 \mathrm{~L} / \mathrm{s}$.

Figures 2 and 3 compare the experimental and numerically predicted flow surface and bed pressure profiles for different discharges and slope transitions. In these figures the nondimensional flow surface elevation and bed pressure at any section, $\eta / H_{c}$ and $p_{b} /\left(\rho g H_{c}+Z_{b} / H_{c}\right) \quad\left(H_{c}=\right.$ critical depth) respectively are shown versus the normalised distance, $x / H_{c}$. Figures 2 and 3 clearly show that the predicted flow surface profiles reveal very satisfactory agreement with the
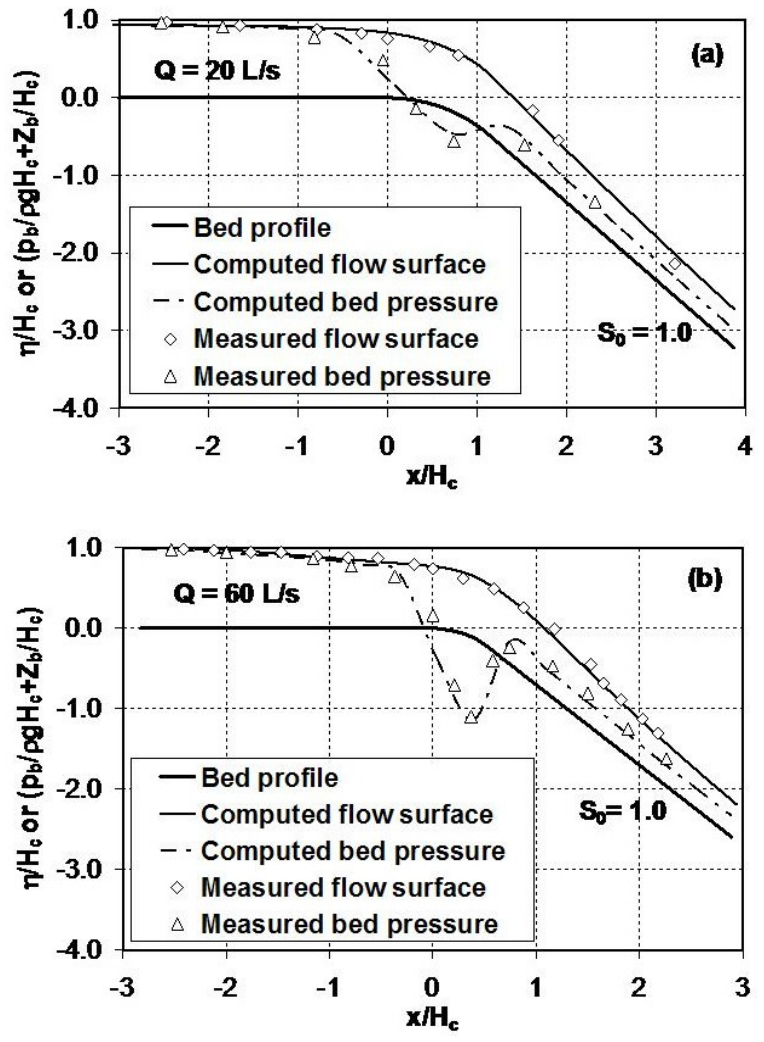

Fig. 2. Comparison of computed flow surface and bed pressure profiles with measured data in a channel transition to $45^{\circ}$.
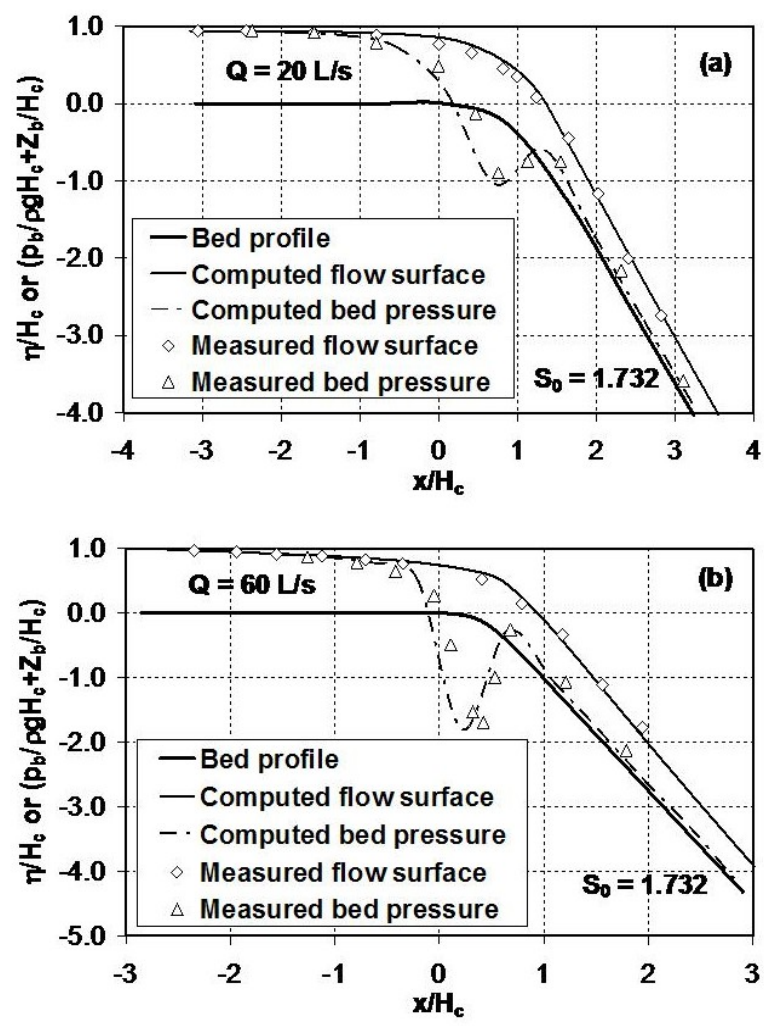

Fig. 3. Comparison of computed flow surface and bed pressure profiles with measured data in a channel transition to $60^{\circ}$.

observed ones. For the $60^{\circ}$ slope transition, the model slightly overestimates the bed pressures in the bed gradient transition region (mean absolute error $=2 \%$ ). It can be seen from Figure 2 
that the model predicts the minimum bed pressure accurately. Downstream of the transition region where the vertical curvature of the streamline is insignificant, the channel steep slope affects the distribution of the pressure (Chaudhry, 2008). In this flow region, the model simulates the bed pressure distributions extremely well. As can be seen from Figures 2 and 3, the bed pressure is considerably below the hydrostatic bed pressure in the region $\left(0<x / H_{c}<1\right)$ where the flow possesses substantial vertical acceleration. This numerical experiment underlines the suitability of a higher-order pressure equation for modelling the pressure distribution of a curvilinear flow in a steeply sloping channel.

The predictions of the present model are further compared in Figure 4 with previous numerical results obtained by Montes (1994) using the Laplace equation based on the potential flow approximation. In the transition region where the effects of the curvature of the bed are substantial, the predictions of the present model compare favourably with the experimental data and show a slight improvement against the numerical results by Montes (1994). In general, the performance of the proposed model in simulating 2-D flows in a channel transition is reasonably good.

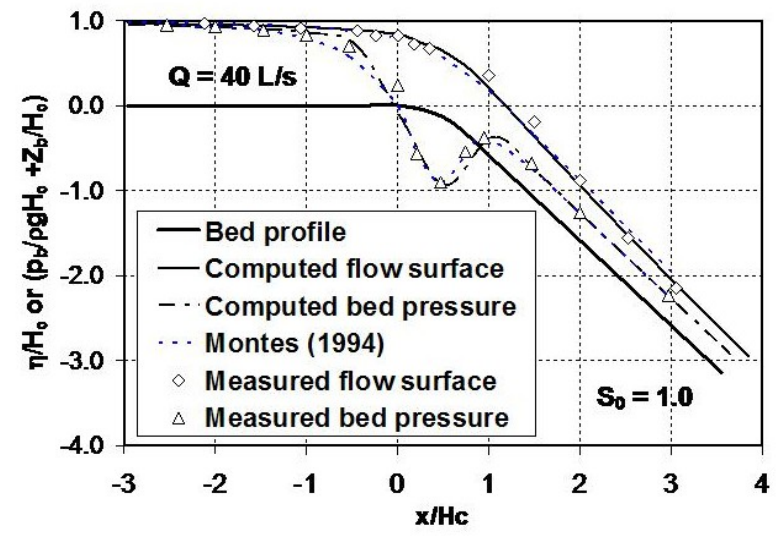

Fig. 4. Comparison of the results of the numerical models with measured data in a channel transition to $45^{\circ}$.

\section{Transcritical flow over a short-crested weir}

The results of the experiments conducted by the author (Zerihun, 2004) were selected to test the prediction of the model. A $7100 \mathrm{~mm}$ long recirculating flume was used to conduct the experiment. Symmetrical trapezoidal profile weirs of 150 $\mathrm{mm}$ height, crest lengths $100 \mathrm{~mm}, 150 \mathrm{~mm}$ and $400 \mathrm{~mm}$ respectively and faces sloped at $26.6^{\circ}$ were tested at different discharges. The flow surface and bed pressure profiles were measured respectively by a point gauge and piezometers. A detailed description of the experimental system can be found in Zerihun (2004).

The computed flow surface and bed pressure profiles for the case of transcritical flow over a trapezoidal profile weir with $H_{0} / L_{w}=1.10$ ( $H_{0}=$ total energy head over the weir crest, $L_{w}=$ weir crest length) are compared with experimental results in Figure 5. The numerical results obtained by Zerihun (2004) using the Fenton's (1996) equations are also plotted. In this figure the non-dimensional flow surface elevation and bed pressure at any section, $\eta / H_{w}$ and $p_{b} / \rho g H_{w} \quad\left(H_{w}=\right.$ weir height) respectively are shown versus the normalised distance from the axis of symmetry of the weir, $x / H_{w}$. The result of
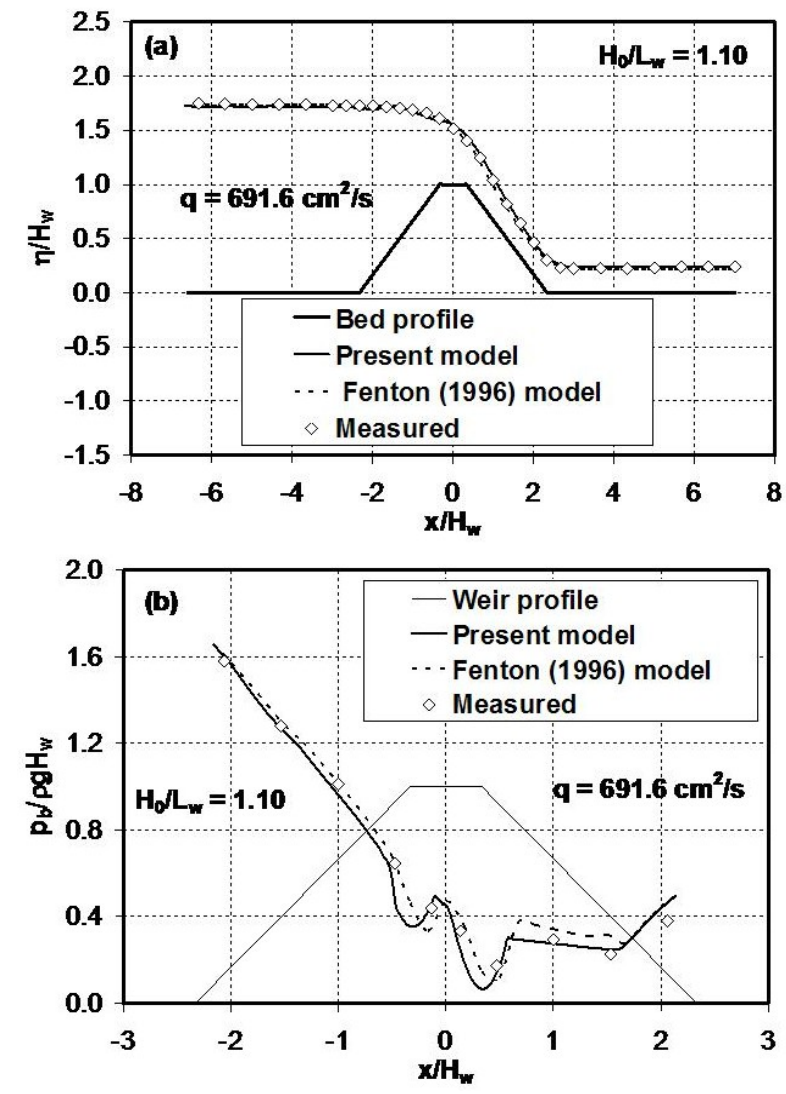

Fig. 5. Comparison of experimental and computed results for flow over a short-crested weir: (a) flow surface profile; (b) bed pressure profile.

the present model for flow surface profile shows excellent agreement with the experimental and previous numerical results, with a maximum error only $1 \%$. Both numerical models predict the steep slope flow surface on the crest of the weir satisfactorily. It can be seen from this figure that the agreement of the predicted bed pressure with experimental data is good for the upstream face of the weir and no significant differences are observed between the computational results of the models. In the supercritical flow region where the flow surface is almost parallel to the downstream face of the weir, the present model simulates the bed pressure profile accurately irrespective of the weir steep slope. However, a minor discrepancy between the experimental and the numerical result of the Fenton's (1996) equations can be seen in this flow region (mean absolute error $=$ $8.1 \%$ ). The results of both models reveal that a local minimum bed pressure occurs near the upstream and downstream edges of the weir crest where the flow possesses a higher dynamic pressure due to the substantial vertical curvatures of the streamline. It should be noted that the experiment cannot capture the bed pressure at these edges due to instrumentation difficulties. In general, the overall level of agreement for the bed pressure is much better with the present model, the mean absolute error being only $2.4 \%$.

\section{Flow over inclined sharp-crested weirs}

This test case was used to examine the nature of the solution of the proposed model for a more complicated flow problem involving dual free surfaces such as flow over an inclined sharp-crested weir. The complete numerical solution of the dual free surfaces flow problem requires the application of both the 
pressure and flow profile equations as well as specifying inflow, internal and outflow boundary conditions. Detailed description of the numerical simulation procedures for this flow problem can be found in Zerihun (2004).

The performance of the model is further assessed by validating the numerical results with test data published by the US Bureau of Reclamation (1948) for strongly curved flows over inclined sharp-crested weirs. Figure 6 compares the computed flow surface profiles using the present model with experimental data and previous numerical results obtained by Khan and Steffler (1996). Similar to the simulation of Khan and Steffler's numerical model, the present model accurately predicts the free surface elevations downstream of the crest section where the streamlines have steep slope and pronounced curvature. Upstream of this section, the present model results correctly reproduce the free surface profiles and compare well with previous numerical results for low and high flow conditions. In general, the overall qualities of the present model results are good for the $45^{\circ}$ and $26.6^{\circ}$ weir slopes test cases.
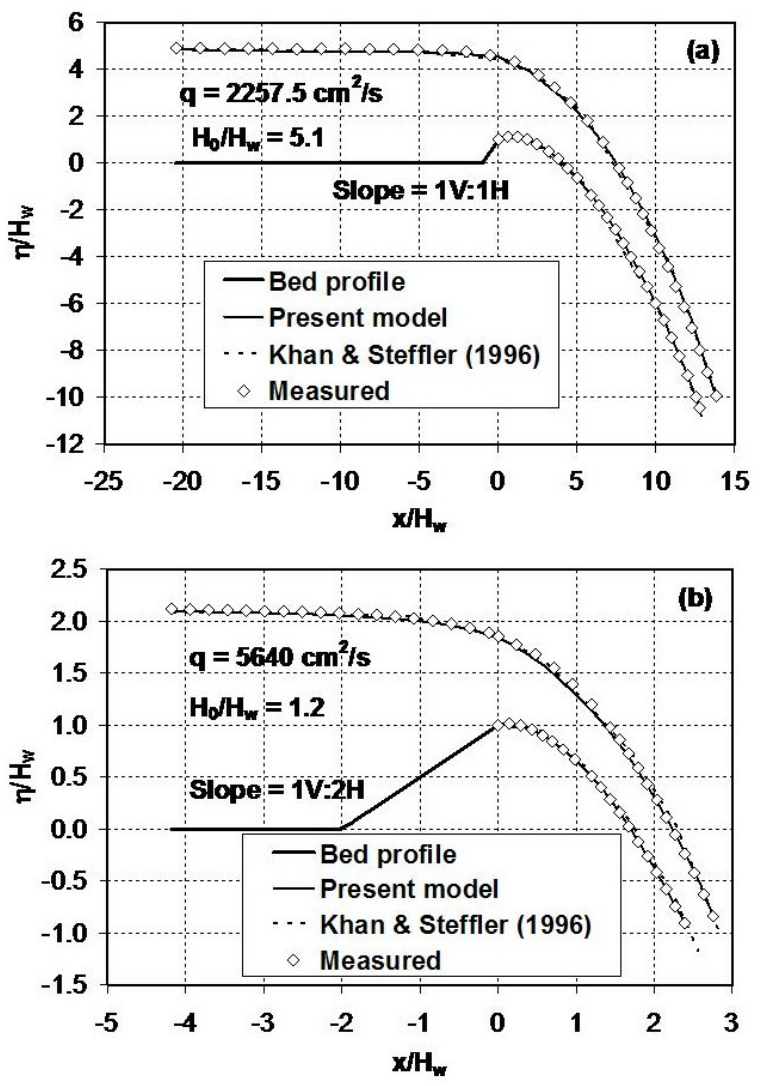

Fig. 6. Comparison of computed and measured flow surface profiles for flow over an inclined sharp-crested weir.

\section{CONCLUSIONS}

A one-dimensional model formulated in the Cartesian coordinate system for open channel flow (Fenton, 1996) was generalised for strongly curved free surface flows in the vertical plane. The proposed model includes the effects of the vertical curvature of the streamline and steep slope, in making the pressure distribution non-hydrostatic, and overcomes the accuracy problem of the Saint-Venant equations when simulating flow situations that involve non-hydrostatic pressure distribution. The significant improvement of the proposed model over previous works is its capacity to model the pressure distribution of curvilinear-streamlined flows in steep slope channels. An implicit finite difference scheme was employed to discretise and solve the model equations. A wide variety of practical free surface flow problems with substantial vertical curvatures of streamline were considered for assessing the suitability of the model. The computational results were also compared with experimental data and earlier numerical results.

For all cases of simulations, the overall qualities of the proposed model results were good and showed good agreement with the experimental data. The predictions of the present model also revealed improvements against the Fenton's (1996) model results and numerical results obtained by Montes (1994) in terms of flow surface and bed pressure profiles. The results of the comparison demonstrate that the proposed model is efficient and applicable to simulate rapidly varied gravity-driven flows with predominant non-hydrostatic pressure distribution effects such as transcritical flows over short-crested weirs and flow with dual free surfaces to a good degree of accuracy. It is concluded that the present model can be applied to other curvilinear flow problems where vertical curvature of the streamline and its effects are important.

\section{REFERENCES}

Abramowitz, M., Stegun, I.A., 1972. Handbook of Mathematical Functions with Formulas, Graphs and Mathematical Tables. 10th edition, Wiley, New York, NY.

Anh, T.N., Hosoda, T., 2007. Depth-averaged model of openchannel flows over an arbitrary 3-D surface and its applications to analysis of water surface profile. J. Hydr. Engrg., 133, 4, 350-360.

Berger, R.C., Carey, G.F., 1998. Free-surface flow over curved surfaces-Part I: Perturbation analysis. Int. J. Numer. Meth. Fluids, 28, 2, 191-200.

Blom, P., Booij, R., 1995. Turbulent free-surface flow over sills. J. Hydr. Res., 33, 5, 663-682.

Castro-Orgaz, O., Hager, W.H., 2009. Curved-streamline transitional flow from mild to steep slopes. J. Hydr. Res., 47, 5, 574-584.

Castro-Orgaz, O., Chanson, H., 2011. Near-critical free-surface flows: real fluid flow analysis. Environ. Fluid Mech., 11, 5, 499-516.

Castro-Orgaz, O., 2013. Potential flow solution for open channel flows and weir crest overflows. J. Irrig. Drain. Engrg., 139, 7, 551-559.

Castro-Orgaz, O., Hager, W.H., 2013. Velocity profile approximations for two-dimensional potential open channel flow. J. Hydr. Res., 51, 6, 645-655.

Castro-Orgaz, O., Chanson, H., 2014. Depth-averaged specific energy in open-channel flow and analytical solution for critical irrotational flow over weirs. J. Irrig. Drain. Engrg., 140, 1, 10.1061/(ASCE)IR.1943-4774.0000666.

Castro-Orgaz, O., Hager, W.H., 2014. One-dimensional modelling of curvilinear free surface flow: generalised Matthew theory. J. Hydr. Res., 52, 1, 14-23.

Chaudhry, M.H., 2008. Open Channel Flow. 2nd edition, Springer Science and Business Media LLC, New York, NY.

Dewals, B.J., Erpicum, S., Archambeau, P., Detrembleur, S., Pirotton, M., 2006. Depth-integrated flow modelling taking into account bottom curvature, J. Hydr. Res., 44, 6, 787-795.

Dressler, R.F., 1978. New nonlinear shallow flow equations with curvature. J. Hydr. Res., 16, 3, 205-220.

Ehrenberger, R., 1929. Versuche über die verteilung der drücke an wehrrücken infolge des abstürzcnden wassers. [Experiments on the distribution of pressures along the face 
of weirs resulting from the impact of the falling water]. Die Wasserwirtschaft, Vienna, 22, 5, 65-72. (In German.)

Fenton, J.D., 1996. Channel flow over curved boundaries and a new hydraulic theory. In: Proc. 10th Congress, APD-IAHR, Langkawi, Malaysia, August 26-29, 2, 266-273.

Ferziger, J.H., Peric, M., 2002. Computational Methods for Fluid Dynamics. 3rd revised edition, Springer-Verlag Berlin Heidelberg, New York, NY.

Fuhrman, D.H., Bingham, H.B., Madsen, P.A., 2005. Nonlinear wave structure interactions with high order Boussinesq model. Coast. Engrg., 52, 8, 655-672.

Ghamry, H.K., Steffler, P.M., 2002. Effect of applying different distribution shapes for velocities and pressure on simulation of curved open channels. J. Hydr. Engrg., 128, 11, 969-982.

Haaland, S.E., 1983. Simple and explicit formulas for the friction factor in turbulent pipe flow. J. Fluids Engrg., 105, 1, 89-90.

Khan, A.A., Steffler, P.M., 1996. Modelling overfalls using vertically averaged and moment equations. J. Hydr. Engrg., 122, 7, 397-402.

Liang, D., Lin, B., Falconer, R.A., 2007. Simulation of rapidly varying flow using an efficient TVD-MacCormack scheme. Int. J. Numer. Meth. Fluids, 53, 5, 811-826.

Matthew, G.D. 1991. Higher order, one-dimensional equations of potential flow in open channels. Proc. Instn. Civ. Eng., London, England, 91, 3, 187-201.

Montes, J.S., 1994. Potential flow solution to 2-D transition from mild to steep slope. J. Hydr. Engrg., 120, 5, 601-621.

Serre, F., 1953. Contribution à l'étude des écoulements permanents et variables dans les canaux [Contribution to the study of steady and unsteady channel flows]. La Houille Blanche, 8, 6-7, 374-388. (In French.)

Steffler, P.M., Jin, Y., 1993. Depth averaged and moment equations for moderately shallow free surface flow. J. Hydr. Res., $31,1,5-17$.

U. S. Bureau of Reclamation, 1948. Studies of crests for overfall dams. Hydraulic Investigations, Bulletin 3, Part VI, Boulder Canyon Project Final Reports, Denver, Colo.

White, F.M., 2003. Fluid Mechanics. 5th edition, McGraw-Hill, New York, NY.

Xia, C., Jin, Y., 2006. Multilayer averaged and moment equations for one-dimensional open-channel flows. J. Hydr. Engrg., 132, 8, 839-849.

Zerihun, Y.T., 2004. A one-dimensional Boussinesq-type momentum model for steady rapidly varied open channel flows. Ph.D. Thesis, Department of Civil and Environmental Engineering, The University of Melbourne, Australia.

Zerihun, Y.T., Fenton, J.D., 2006. One-dimensional simulation model for steady transcritical free surface flows at short length transitions. Adv. Water Resour., 29, 11, 1598-1607.

Zobeyer, H., Steffler, P.M., 2012. Modelling plane openchannel flows by coupled depth-averaged and RANS equations. J. Hydr. Res., 50, 1, 82-88.

\section{NOMENCLATURE}

The following symbols are used in this paper

$A \quad$ flow cross-sectional area $\left(\mathrm{L}^{2}\right)$

$a_{n} \quad$ normal acceleration $\left(\mathrm{LT}^{-2}\right)$

$B \quad$ width of the channel (L)

$F \quad$ the Froude number (-)

$G \quad$ body force per unit mass $\left(\mathrm{LT}^{-2}\right)$

$g \quad$ acceleration of gravity $\left(\mathrm{LT}^{-2}\right)$

$h \quad$ step size (L)

$h_{s} \quad$ vertical distance above the bed (L)

$H \quad$ depth of flow in vertical direction (L)

$H_{c} \quad$ critical depth (L)

$m$ total number of computational nodes (-)

$n \quad$ curvilinear coordinate along normal (L)

$p \quad$ pressure $\left(\mathrm{ML}^{-1} \mathrm{~T}^{-2}\right)$

$p_{b} \quad$ bed pressure $\left(\mathrm{ML}^{-1} \mathrm{~T}^{-2}\right)$

$p_{0} \quad$ hydrostatic pressure $\left(\mathrm{ML}^{-1} \mathrm{~T}^{-2}\right)$

$Q \quad$ discharge $\left(\mathrm{L}^{3} \mathrm{~T}^{-1}\right)$

$R \quad$ radius of curvature $(\mathrm{L})$

$s \quad$ curvilinear coordinate along streamline (L)

$S_{f} \quad$ friction slope (-)

$S_{H} \quad$ free surface slope (-)

$S_{0} \quad$ bed slope (-)

$t$ time (T)

$u \quad$ horizontal velocity $\left(\mathrm{LT}^{-1}\right)$

$V \quad$ tangential velocity $\left(\mathrm{LT}^{-1}\right)$

$w \quad$ vertical velocity $\left(\mathrm{LT}^{-1}\right)$

$x, y$ and $z \quad$ Cartesian coordinates (L)

$Z_{b} \quad$ channel bed elevation (L)

$Z_{b}^{\prime}, Z_{b}^{\prime \prime}, Z_{b}^{\prime \prime \prime}$ derivatives of the bed profile $\left(-, \mathrm{L}^{-1}, \mathrm{~L}^{-2}\right)$

$\beta \quad$ Boussinesq coefficient (-)

$\delta H_{j} \quad$ correction depth at node $j$ (L)

$\eta \quad$ elevation of the free surface (L)

$\theta \quad$ angle of streamline with horizontal (rad)

$\kappa \quad$ streamline curvature $\left(\mathrm{L}^{-1}\right)$

$\kappa_{b} \quad$ bed curvature $\left(\mathrm{L}^{-1}\right)$

$\kappa_{s} \quad$ curvature of the free surface $\left(\mathrm{L}^{-1}\right)$

$\rho \quad$ density of the fluid $\left(\mathrm{ML}^{-3}\right)$

$\omega$ dynamic pressure correction term $\left(\mathrm{L}^{-1}\right)$

Received 8 October 2015 Accepted 15 April 2016 